\title{
THE PROOF THAT A GAME MAY NOT HAVE A SOLUTION( $\left.{ }^{1}\right)$
}

\author{
BY \\ WILLIAM F. LUCAS
}

1. Introduction. In 1944 von Neumann and Morgenstern [3] introduced a theory of solutions (stable sets) for $n$-person games in characteristic function form. It has been shown that solutions do exist for several large classes of games, and it had been conjectured that every game has at least one solution. However, the author [2] recently described a ten-person game which has no solution. This paper gives a detailed proof that there is no solution for this game. The essential definitions for an $n$-person game will be reviewed before the counterexample and the proof are given.

2. Definitions. An $n$-person game is a pair $(N, v)$ where $N=\{1,2, \ldots, n\}$ is a set of $n$ players labeled $1,2, \ldots, n$ and where $v$ is a real valued characteristic function on $2^{N}$, i.e., $v$ assigns a real number $v(S)$ to each nonempty subset $S$ of $N$ and $v(\varnothing)=0$ for the empty set $\varnothing$. Intuitively, the number $v(S)$ represents the value (wealth, power) of the coalition $S$. The set of imputations is

$$
A=\left\{x: \sum_{h \in N} x_{h}=v(N) \text { and } x_{h} \geqq v(\{h\}) \text { for all } h \in N\right\}
$$

where $x=\left(x_{1}, x_{2}, \ldots, x_{n}\right)$ is a vector with real components. Each imputation $x$ represents a realizable distribution of the wealth among the players; player $h$ receives the amount $x_{h}$.

The players in a coalition $S$ will prefer imputation $x$ to $y$ if each of them receives more in $x$ and if they can effect $x$. If $x$ and $y \in A$ and $S$ is a nonempty subset of $N$, then $y$ dominates $x$ via $S$, denoted $y \operatorname{dom}_{S} x$, means

$$
y_{h}>x_{h} \text { for all } h \in S
$$

and

$$
\sum_{h \in S} y_{h} \leqq v(S)
$$

If there exists an $S$ such that $y \operatorname{dom}_{S} x$, then one says that $y$ dominates $x$ and denotes this by $y \operatorname{dom} x$. For any $y \in A$ and $Y \subset A$ define the following dominions:

$$
\begin{aligned}
\operatorname{Dom}_{S} y & =\left\{x \in A: y \operatorname{dom}_{S} x\right\}, \\
\operatorname{Dom}_{y} & =\{x \in A: y \operatorname{dom} x\}, \\
\operatorname{Dom}_{S} Y & =\bigcup_{y \in Y} \operatorname{Dom}_{S} y, \\
\operatorname{Dom} Y & =\bigcup_{y \in Y} \operatorname{Dom} y,
\end{aligned}
$$

Received by the editors January 16, 1968.

(1) This research was supported by the United States Air Force under Project RAND. 
and the inverse dominions:

$$
\begin{aligned}
\operatorname{Dom}^{-1} y & =\{z \in A: z \operatorname{dom} y\} \\
\operatorname{Dom}^{-1} Y & =\bigcup_{y \in Y} \operatorname{Dom}^{-1} y .
\end{aligned}
$$

To simplify the notation in (2) let

$$
y(S)=\sum_{h \in S} y_{h}
$$

Also, expressions such as $x(\{1,4,7,9\})$ will be shortened to $x(1,4,7,9)$ or $x(1479)$ throughout the paper.

A subset $K$ of $A$ is a solution for a game if

$$
K \cap \operatorname{Dom} K=\varnothing
$$

and

$$
K \cup \operatorname{Dom} K=A \text {. }
$$

If $K^{\prime} \subset X \subset A$, then $K^{\prime}$ is a solution for $X$ means that

$$
K^{\prime} \cap \operatorname{Dom} K^{\prime}=\varnothing
$$

and

$$
K^{\prime} \cup \operatorname{Dom} K^{\prime} \supset X .
$$

The core of the game $(N, v)$ is

$$
C=\{x \in A: x(S) \geqq v(S) \text { for all } S \subset N\} .
$$

The core is a convex polyhedron (possibly empty). It is clear that for any solution $K$, $C \subset K$ and $K \cap \operatorname{Dom} C=\varnothing$.

3. Example. A game which has no solution is $(N, v)$ where

$$
N=\{1,2,3,4,5,6,7,8,9,10\}
$$

and $v$ is given by:

$$
\begin{aligned}
& v(N)=5, v(\{1,3,5,7,9\})=4, \\
& v(\{1,2\})=v(\{3,4\})=v(\{5,6\})=v(\{7,8\})=v(\{9,10\})=1, \\
& v(\{3,5,7,9\})=v(\{1,5,7,9\})=v(\{1,3,7,9\})=3, \\
& v(\{3,5,7\})=v(\{1,5,7\})=v(\{1,3,7\})=2, \\
& v(\{3,5,9\})=v(\{1,5,9\})=v(\{1,3,9\})=2, \\
& v(\{1,4,7,9\})=v(\{3,6,7,9\})=v(\{5,2,7,9\})=2, \\
& v(S)=0 \text { for all other } S \subset N .
\end{aligned}
$$

The set of imputations for this game is

$$
A=\left\{x: x(N)=5 \text { and } x_{h} \geqq 0 \text { for all } h \in N\right\} .
$$


It is helpful to introduce the five-dimensional hypercube

$$
B=\{x \in A: x(1,2)=x(3,4)=x(5,6)=x(7,8)=x(9,10)=1\},
$$

and the following six vertices of $B$ :

$$
\begin{array}{ll}
c^{0}=(1,0,1,0,1,0,1,0,1,0), & c^{2}=(0,1,1,0,1,0,1,0,1,0), \\
c^{4}=(1,0,0,1,1,0,1,0,1,0), & c^{6}=(1,0,1,0,0,1,1,0,1,0), \\
c^{8}=(1,0,1,0,1,0,0,1,1,0), & c^{10}=(1,0,1,0,1,0,1,0,0,1) .
\end{array}
$$

One can show that the core of this game is the convex hull of the six imputations $c^{h}(h=0,2,4,6,8,10)$. For the proofs in this paper it is sufficient to prove that $C$ contains this convex hull. This can be done by simply checking that each of these $c^{h}$ satisfies all of the inequalities $x(S) \geqq v(S)$ in the definition of $C$. It is also easy to check that any $x \in B$ which satisfies $x(13579) \geqq 4=v(\{1,3,5,7,9\})$ will be in $C$, and thus

$$
C=\{x \in B: x(13579) \geqq 4\} .
$$

4. Partition of $A$. To prove that this game has no solution, it is necessary to define several regions in $A$. It will be assumed in these definitions and throughout the remainder of this paper (unless otherwise specified) that the variable indices $i, j, k, p, q$, and $r$ take on the values

$$
(i, j, r, k)=(1,3,4,5),(3,5,6,1), \text { and }(5,1,2,3)
$$

and

$$
(p, q)=(7,9) \text { and }(9,7) \text {. }
$$

For example, an expression containing the subscript $i$ stands for the three expressions where $i=1,3$, and 5; an expression containing the indices, $i, j$, and $k$ represents the three expressions where the ordered triple $(i, j, k)=(1,3,5),(3,5,1)$, and $(5,1,3)$; and an expression containing $p$ represents the two expressions where $p=7$ and 9 . The letter $h$ will continue as a free index which is defined whenever it is used.

Using these conventions on the variable indices, define the following twelve subsets of $B$ :

$$
\begin{aligned}
E_{i} & =\left\{x \in B: x_{j}=x_{k}=1, x_{i}<1, x(79)<1\right\}, \\
E & =E_{1} \cup E_{2} \cup E_{3}, \\
F_{j k} & =\left\{x \in B: x_{j}=x_{k}=1,1 \leqq x(79)\right\}-C, \\
F_{p} & =\left\{x \in B: x_{p}=1, x_{q}<1, x(35 q) \geqq 2, x(51 q) \geqq 2, x(13 q) \geqq 2\right\}-C, \\
F_{79} & =\left\{x \in B: x_{7}=x_{9}=1\right\}-C, \\
F_{135} & =\left\{x \in B: x_{1}=x_{3}=x_{5}=1\right\}-C, \\
F & =F_{35} \cup F_{51} \cup F_{13} \cup F_{7} \cup F_{9} \cup F_{79} \cup F_{135} .
\end{aligned}
$$

Figure 1 pictures the traces of these various regions as they appear in some of the (three-dimensional) cubical traces of the five-dimensional hypercube $B$. Figure 2 illustrates a wedge $E_{j} \cup F_{135}$. 

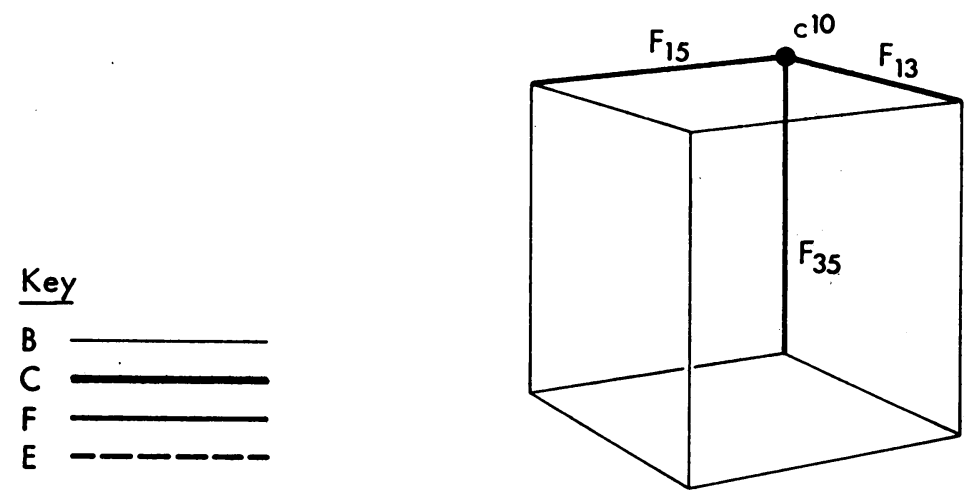

$$
x_{7}=1, x_{8}=0, x_{9}=0, x_{10}=1
$$

In each cube

Top face: $\quad x_{1}=1, x_{2}=0$

Bottom face: $\quad x_{1}=0, x_{2}=1$

Right face: $\quad x_{3}=1, x_{4}=0$

Left face: $\quad x_{3}=0, x_{4}=1$

Back face: $\quad x_{5}=1, x_{6}=0$

Front face: $\quad x_{5}=0, x_{6}=1$

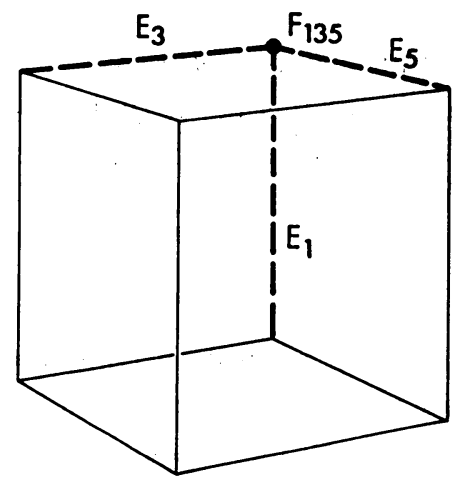

$x_{7}=\frac{1}{2}, x_{8}=\frac{1}{2}, x_{9}=0, x_{10}=1$

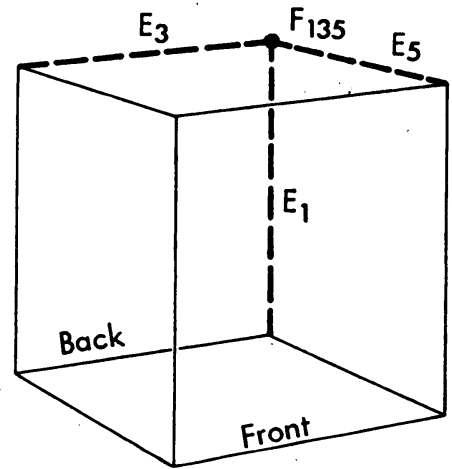

$x_{7}=0, x_{8}=1, x_{9}=0, x_{10}=1$ 

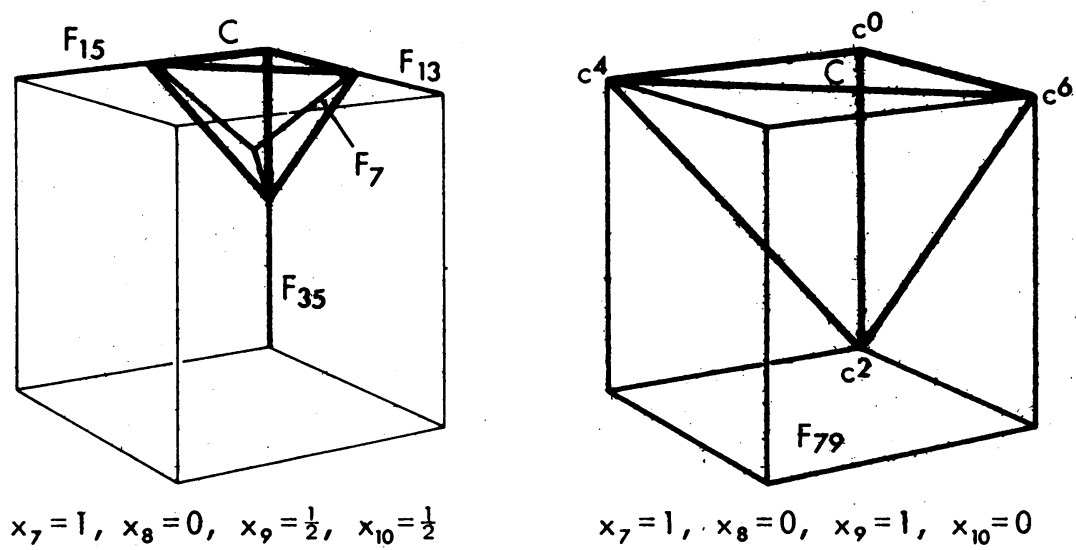

$$
x_{7}=1, x_{8}=0, x_{9}=1, x_{10}=0
$$
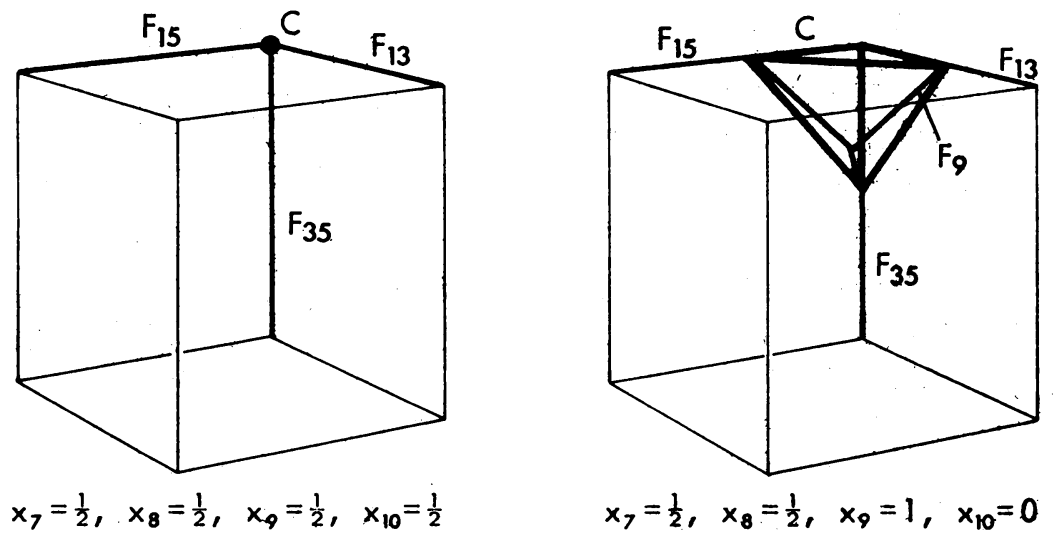

$$
x_{7}=\frac{1}{2}, x_{8}=\frac{1}{2}, x_{9}=1, x_{10}=0
$$
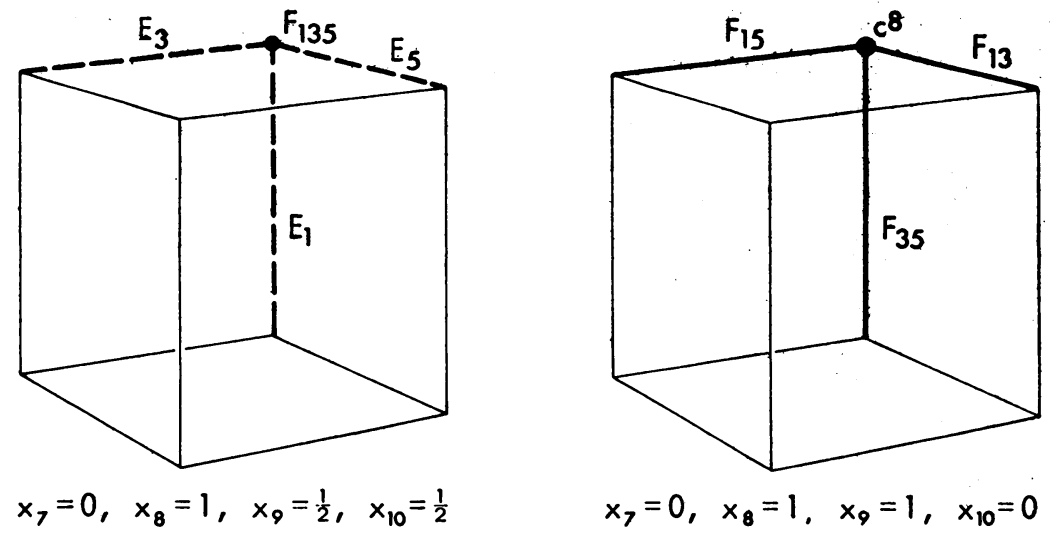

$x_{7}=0, x_{8}=1, x_{9}=\frac{1}{2}, x_{10}=\frac{1}{2}$

Figure 1. Traces in $B$ of $C, F$ and $E$ for constant $x_{7}, x_{8}, x_{9}$, and $x_{10}$. 


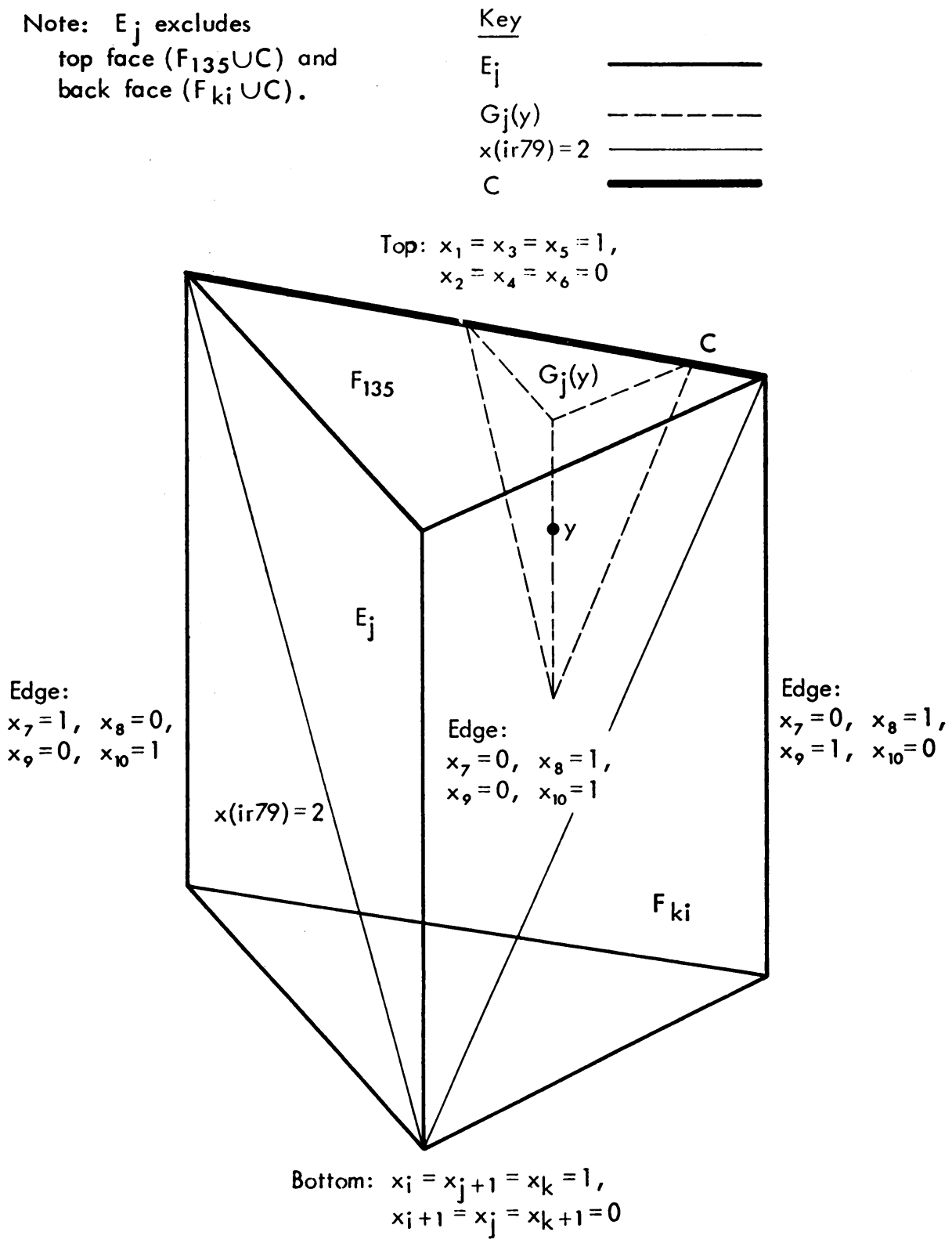

FIGURE 2. Regions $E_{j}, F_{135}$, and $G_{j}(y)$. 
The regions $A-B, B-(C \cup E \cup F), C, E$, and $F$ form a partition of $A$. This is obvious if one verifies that $C \cap E=\varnothing$ and $E \cap F=\varnothing$. However, if $x \in E$ then $x_{h} \leqq 1(h=1,3$, and 5) and $x(79)<1$. Thus $x(13579)<4$ and so $C \cap E=\varnothing$. From the definitions, $E \cap F_{135}=\varnothing$, and if $y \in F-F_{135}$ then $y(79) \geqq 1$. Thus $E \cap F=\varnothing$.

To prove that this game has no solution it is sufficient to prove that

(I) Dom $C \supset[A-B] \cup[B-(C \cup E \cup F)]$,

(II) $E \cap \operatorname{Dom}(C \cup F)=\varnothing$, and

(III) there exists no solution $K^{\prime}$ for $E$.

5. Dominion of the core. First, one must show that Dom $C \supset A-B$. Pick $x \in A-B$, i.e., consider any $x$ with $x_{h} \geqq 0$ for all $h \in N, \sum_{h \in N} x_{h}=5$, and $x(h-1, h)$ $\neq 1$ for some $h=2,4,6,8$ or 10 . It is clear that there exists an $h=2,4,6,8$, or 10 such that $x(h-1, h)<1$. For this $h$ let $y=\left(x_{h-1}+e\right) c^{0}+\left(x_{h}+e\right) c^{h}$ where $2 e=1$ $-x_{h-1}-x_{h}>0$. Then $y \in C$, and $y \operatorname{dom}_{\{h-1, h\}} x$, since $y_{h-1}=x_{h-1}+e>x_{h-1}$, $y_{h}=x_{h}+e>x_{h}$, and $y(h-1, h)=x(h-1, h)+2 e=1$. In summary, the closed line segment joining $c^{0}$ and $c^{h}$ is contained in $C$ and its dominion contains any $x \in A$ with $x(h-1, h)<1$. Therefore, Dom $C \supset A-B$, and thus one needs only to consider the region $B$ when looking for solutions to this game.

Next, one must prove that Dom $C \supset B-(C \cup E \cup F)$. Pick any $x \in B$ $-(C \cup E \cup F)$. It follows from the definitions of $B, C, E$, and $F$ that $x$ must satisfy the conditions

$$
\begin{gathered}
0 \leqq x_{h} \leqq 1 \text { for all } h \in N, \\
x(13579)<4, \\
x_{j}<1, x_{k}<1 \quad \text { for }(j, k)=(3,5),(5,1), \text { or }(1,3), \\
x_{q}<1,
\end{gathered}
$$

and either

$$
x_{p}<1
$$

or

$$
x_{p}=1 \text { and either } x(35 q)<2, x(51 q)<2 \text {, or } x(13 q)<2
$$

where $(p, q)=(7,9)$ or $(9,7)$ in the relations $(8),(9)$, and $\left(9^{\prime}\right)$. It follows that if $x \in B-(C \cup E \cup F)$ then $x \in B$ and either

(i) $x(13579)<4$ and $x_{h}<1$ for $h=1,3,5$, 7, and 9,

(ii) $x(13579)<4, x_{i}=1$, and $x_{h}<1$ for $h=j, k$, 7, and 9, or

(iii) $x(13579)<4, x_{i} \leqq 1, x_{p}=1, x_{h}<1$ for $h=j, k$, and $q$, and either $x(j k q)<2$ or $x($ imq $)<2$ for $m=j$ or $k$

where $(i, j, k)=(1,3,5),(3,5,1)$, or $(5,1,3)$, and $(p, q)=(7,9)$ or $(9,7)$.

Each of these three cases will now be considered.

In case (i), one can pick $y \in B$ such that $y(13579)=4$ and $1 \geqq y_{h}>x_{h}$ for $h=1,3,5,7$, and 9 . Observe that $y(2,4,6,8,10)=5-y(1,3,5,7,9)=1$; 
$y(\{2,4,6,8,10\}-\{h\})=1-y_{h}=y_{h-1}$ for $h=2,4,6,8$, and 10; and thus $y=\sum_{h} y_{h} c^{h}$ where $h=2,4,6,8$, and 10 . Therefore, $y$ is in the convex hull of $c^{2}, c^{4}, c^{6}, c^{8}$, and $c^{10}$, and $y \operatorname{dom}_{\{2,4,6,8,10\}} x$. In summary, the convex hull of the $c^{h}(h=2,4,6,8$, and 10) is contained in $C$ and its dominion contains all $x \in B$ which satisfy.(i).

In case (ii), $x(j k 79)<3$, since $x_{i}=1$ and $x(13579)<4$. In this case one can pick $y \in B$ such that $y(j k 79)=3, y_{i}=1$, and $1 \geqq y_{h}>x_{h}$ for $h=j, k, 7$, and 9. Observe that $y(j+1, k+1,8,10)=5-y(i, i+1)-y(j, k, 7,9)=1 ; y(\{j+1, k+1,8,10\}-\{h\})$ $=1-y_{h}=y_{h-1}$ for $h=j+1, k+1,8$, and 10 ; and thus $y=\sum_{h} y_{h} c^{h}$ where $h=j+1$, $k+1,8$, and 10. Therefore, $y$ is in the convex hull of $c^{j+1}, c^{k+1}, c^{8}$, and $c^{10}$, and $y \operatorname{dom}_{(j, k, 7,9)} x$. In summary, the convex hull of the $c^{h}(h=j+1, k+1,8$, and 10) is contained in $C$ and its dominion contains all $x \in B$ which satisfies (ii).

Consider case (iii) and first assume that $x(j k q)<2$. One can pick $y \in B$ such that $y(j k q)=2, y_{\imath}=1, y_{p}=1$, and $1 \geqq y_{h}>x_{h}$ for $h=j, k$, and $q$. Observe that

$$
y(j+1, k+1, q+1)=5-y(i, i+1)-y(j, k, q)-y(p, p+1)=1 ;
$$

$y(\{j+1, k+1, q+1\}-\{h\})=1-y_{h}=y_{h-1}$ for $h=j+1, k+1$, and $q+1$; and thus $y=\sum_{h} y_{h} c^{h}$ when $h=j+1, k+1$, and $q+1$. Therefore, $y$ is in the convex hull of $c^{j+1}, c^{k+1}$, and $c^{q+1}$, and $y \operatorname{dom}_{\{j, k, q\}} x$. In summary, the convex hull of $c^{h}(h=j+1$, $k+1$, and $q+1$ ) is contained in $C$ and its dominion contains all $x \in B$ which satisfy (iii) and $x(j k q)<2$.

Consider case (iii) and now assume that $x($ imq $)<2$ for $m=j$ or $k$. If $x_{i}<1$ then the proof for this case is the same as in the preceding paragraph only $i, m$, and $q$ now take the place of $j, k$, and $q$. If $x_{i}=1$ and $m=j$ [or $m=k$ ] then $x_{k}<1=x_{i}$ [or $x_{j}$ $<1=x_{i}$ ], and thus $x(j k q)<x(i m q)<2$; and this case becomes the same case as in the preceding paragraph,

This completes the proof that Dom $C \supset B-(C \cup E \cup F)$, and thus condition (I) has been verified.

6. Domination into the region $E$. The next step is to prove condition (II), i.e., $E \cap \operatorname{Dom}(C \cup F)=\varnothing$. This will now be done by considering several cases. In the following cases return to the convention that any expression involving some of the indices $i, j, r$, and $k$ will continue to represent the three expressions where $(i, j, r, k)$ $=(1,3,4,5),(3,5,6,1)$, and $(5,1,2,3)$.

First, observe that

$$
A \cap \operatorname{Dom}_{S} A=\varnothing
$$

when $S=N$ and when $v(S)=0$.

Second, note that

$$
E \cap \operatorname{Dom}_{\{h-1, h\}} A=\varnothing \quad \text { for all } h=2,4,6,8 \text {, and } 10
$$

since $E \subset B$ and $x(h-1, h)=1=v(\{h-1, h\})$ for all $x \in B$.

Third, one can show that

$$
E_{i} \cap \operatorname{Dom}_{S} B=\varnothing \quad \text { for all } S \neq\{i, r, 7,9\} .
$$


If $x \in E_{i}$ then $x_{j}=x_{k}=1$, and if $y \in B$ then $y_{h} \leqq 1$ for all $h \in N$. Thus $y$ cannot dominate $x$ via any $S$ which contains $j$ or $k$. This remark along with (10) and (11) proves (12).

Fourth, one proves that

$$
E \cap \operatorname{Dom}_{\{i, r, 7,9\}} C=\varnothing .
$$

If $y \in C$ and $\operatorname{Dom}_{\{i, r, 7,9\}} y \neq \varnothing$, then $0 \leqq y_{h} \leqq 1$ for all $h \in N, y(13579) \geqq 4$, and $y\left(\right.$ ir 79) $\leqq 2$. This implies that $y_{j}=y_{k}=1$ and $y_{r}=0$, and thus $\operatorname{Dom}_{\{i, r, 7,9\}} y=\varnothing$ and (13) holds.

Fifth, one can show that

$$
E \cap \operatorname{Dom}_{\{i, r, 7,9\}} F=\varnothing .
$$

If $y \in F$ and $\operatorname{Dom}_{\{i, r, 7,9\}} y \neq \varnothing$, then $y_{r}>0$ and $y(i r 79) \leqq 2$. However, if one considers the different parts of $F$, he can show that one of these latter two inequalities fails to hold. If $y \in F_{79}$ then $y(79)=2$, and thus $y_{i}$ and $y_{r}=0$ or $y\left(\right.$ ir 79) $>2$. If $y \in F_{135}$ then $y_{1}=y_{3}=y_{5}=1$, and thus $y_{2}=y_{4}=y_{6}=0$, i.e., $y_{r}=0$. If $y \in F_{j k}$, then $y_{j}=y_{k}=1$, and thus $y_{j+1}=y_{k+1}=0$, i.e., $y_{r}=0$. If $y \in F_{i h}(h=j$ or $k)$, then $y_{i}=1$ and $y(79) \geqq 1$, and thus $y_{r}=0$ or $y\left(\right.$ ir 79) $>2$. If $y \in F_{p}$ (recall that the pair $(p, q)=(7,9)$ and $\left.(9,7)\right)$, then $y_{p}=1$ and $y(i h q) \geqq 2$ for $h=j$ and $k$; and thus $y(i q) \geqq y(i h q)-1 \geqq 1$, and so $y_{r}=0$ or $y(\operatorname{ir} 79)=y($ irpq $)>2$.

The conditions (10) through (14) are sufficient to prove (II). Although it will not be done in this paper, one could prove in addition that $F \cap$ Dom $(C \cup E \cup F)=\varnothing$. This would imply that $C \cup F$ is contained in any solution $K$ for this game.

7. Domination within the region $E$. In order to prove condition (III), i.e., that there exists no solution $K^{\prime}$ for $E$, one must consider possible domination between imputations in $E$. However, (10), (11), and (12) imply that only domination via coalitions of the type $\{i, r, 7,9\}$ need be considered. Furthermore,

$$
E_{i} \cap \operatorname{Dom}_{\{i, r, 7,9\}}\left(E_{i} \cup E_{k}\right)=\varnothing,
$$

because if $y \in E_{i} \cup E_{k}$ then $y_{j}=1$, and thus $y_{j+1}=y_{r}=0$. Therefore, the only possible domination within $E$ is illustrated by the diagram:

$$
E_{5} \underset{\{3,6,7,9\}}{\longrightarrow} E_{3} \underset{\{1,4,7,9\}}{\longrightarrow} E_{1} \underset{\{5,2,7,9\}}{\longrightarrow} E_{5} .
$$

Next, one can consider what a typical element in $E$ will dominate and what will dominate it. First, define the three regions

$$
G_{j}(y)=\left\{x \in E_{j}: x_{7}>y_{7}, x_{9}>y_{9}, \text { and } x(\text { ir } 79) \leqq 2\right\}
$$

where $y$ can be any element in $E$. (See Figure 2.) It is clear that the $G_{j}(y)$ are nonempty. If $y \in E_{j}$ and $y\left(\right.$ ir 79) $>2$, then $E \cap$ Dom $y=\varnothing$; and if $y \in E_{j}$ and $y($ ir 79) $\leqq 2$, then

$$
E \cap \operatorname{Dom} y=\left\{x \in E_{i}: x_{7}<y_{7} \text { and } x_{9}<y_{9}\right\}
$$


since $y_{i}=1>x_{i}$ and $y_{r}=y_{j+1}>0=x_{j+1}=x_{r}$. Similarly, one sees that if $y \in E_{i}$ then

$$
E \cap \operatorname{Dom}^{-1} y=\left\{x \in E_{j}: x_{7}>y_{7}, x_{9}>y_{9} \text {, and } x(\text { ir79) } \leqq 2\}=G_{j}(y)\right. \text {. }
$$

One can also show that for any $y \in E$, Dom $G_{i}(y) \supset G_{k}(y)$ and

$$
E \cap \operatorname{Dom}^{-1} G_{k}(y)=G_{i}(y) .
$$

Finally, one can assume that there exists a solution $K^{\prime}$ for $E$ and arrive at a contradiction. Clearly $K^{\prime} \neq \varnothing$. Pick an arbitrary $z \in K^{\prime}$ and assume that $z \in E_{j}$ where $j=1,3$, or 5 . For the remainder of this section $(i, j, k)$ will always refer to one particular triple $(1,3,5),(3,5,1)$, or $(5,1,3)$. Condition $\left(3^{\prime}\right)$ implies that $K^{\prime}$ $\cap \operatorname{Dom}^{-1} z=\varnothing$, and thus

$$
K^{\prime} \cap G_{k}(z)=\varnothing
$$

Thus (4'), (15), and (16) imply that

$$
K^{\prime} \cap G_{i}(z) \neq \varnothing
$$

One can therefore pick a $z^{\prime} \in K^{\prime} \cap E_{i}$ with $z_{7}^{\prime}>z_{7}$ and $z_{9}^{\prime}>z_{9}$. Repeating the argument up to (17) for $z^{\prime}$, one obtains

$$
K^{\prime} \cap G_{k}\left(z^{\prime}\right) \neq \varnothing .
$$

Since $G_{k}\left(z^{\prime}\right) \subset G_{k}(z)$, conditions (16) and (18) give the desired contradiction. Therefore, (III) is verified, and this completes the proof that this game has no solution.

8. Superadditivity. An arbitrary game $(N, v)$ is said to have a superadditive characteristic function if $v\left(S_{1} \cup S_{2}\right) \geqq v\left(S_{1}\right)+v\left(S_{2}\right)$ whenever $S_{1} \cap S_{2}=\varnothing$. The classical theory of games assumed a superadditive $v$. The particular game $(N, v)$ described in this paper does not have a superadditive $v$. However, one can use a method of Gillies [1, pp. 68-69] to extend this game to a game $\left(N, v^{\prime}\right)$ with a superadditive $v^{\prime}$. For every $S \subset N$ define

$$
v^{\prime}(S)=\max \sum_{h=1}^{m} v\left(S_{h}\right)
$$

where this maximum is taken over all partitions $\left\{S_{1}, S_{2}, \ldots, S_{m}\right\}$ of $S$. The resulting $v^{\prime}$ is superadditive. One can also check that for this particular game, $v^{\prime}(S)=v(S)$ for all $S \subset N$ which have $v(S)>0$. In particular $v^{\prime}(N)=v(N)$ and thus the set $A$ remains the same. One can also show (see Gillies) that if $y \operatorname{dom} x$ in the game $\left(N, v^{\prime}\right)$, then $y$ dom $x$ in the game $(N, v)$. Thus the game $\left(N, v^{\prime}\right)$ has the same core and solutions as $(N, v)$. Therefore, the game $\left(N, v^{\prime}\right)$ has a superadditive $v^{\prime}$ and it has no solution.

9. Acknowledgment. The author is grateful to L. S. Shapley for many helpful discussions. 


\section{REFERENCES}

1. D. B. Gillies, Solutions to general non-zero-sum games, Ann. of Math. Studies, No. 40, Princeton Univ. Press, Princeton, N. J., 1959, pp. 47-85.

2. W. F. Lucas, A game with no solution, Bull. Amer. Math. Soc. 74 (1968), 237-239.

3. J. von Neumann and O. Morgenstern, Theory of games and economic behavior, Princeton Univ. Press, Princeton, N. J., 1944.

THE RAND CORPORATION,

Santa Monica, California 